\title{
Positive solutions for nonlinear elliptic equations with fast increasing weights
}

\section{Florin Catrina}

Department of Mathematics and Computer Science, St. John's University, Queens, NY 11439, USA

(catrinaf@stjohns.edu)

Marcelo Furtado

Departamento de Matemática, Universidade de Brasília, CEP 70910-900, Brasília, DF, Brazil (mfurtado@unb.br)

\section{Marcelo Montenegro}

Departamento de Matemática, Universidade Estadual de Campinas, IMECC, Caixa Postal 6065, CEP 13083-970, Campinas, SP, Brazil (msm@ime.unicamp.br)

(MS received 6 July 2006; accepted 8 November 2006)

We find positive rapidly decaying solutions for the equation

$$
-\operatorname{div}(K(x) \nabla u)=K(x) u^{2^{*}-1}+\lambda K(x)|x|^{\alpha-2} u
$$

in $\mathbb{R}^{N}$, where $N \geqslant 3$, the nonlinearity is given by the critical Sobolev exponent $2^{*}=2 N /(N-2)$, the weight is $K(x)=\exp \left(\frac{1}{4}|x|^{\alpha}\right), \alpha \geqslant 2$ and $\lambda$ is a parameter.

\section{Introduction}

We study the equation

$$
-\operatorname{div}(K(x) \nabla u)=K(x) u^{2^{*}-1}+\lambda K(x)|x|^{\alpha-2} u, \quad u>0 \in \mathbb{R}^{N},
$$

where $N \geqslant 3$, the nonlinearity is given by the critical Sobolev exponent $2^{*}=$ $2 N /(N-2)$, the weight is $K(x)=\exp \left(\frac{1}{4}|x|^{\alpha}\right), \alpha \geqslant 2$ and $\lambda$ is a parameter. According to the function space in which we seek solutions, $u$ is forced to decrease sufficiently fast to infinity.

As in [12], for $\alpha=2$ and $\lambda=(N-2) /(N+2)$, equation (1.1) occurs when one tries to find self-similar solutions

$$
v(x, t)=t^{(2-N) /(N+2)} u\left(x t^{-1 / 2}\right)
$$

to the parabolic equation

$$
v_{t}-\Delta v=|v|^{4 /(N-2)} v, \quad \mathbb{R}^{N} \times(0,+\infty) .
$$

Notice that (1.1) is equivalent to

$$
-\Delta u-\frac{1}{4} \alpha|x|^{\alpha-2}(x \cdot \nabla u)=|u|^{2^{*}-2} u+\lambda|x|^{\alpha-2} u .
$$

(C) 2007 The Royal Society of Edinburgh 
Equation (1.1) was treated in [11] in the case $\alpha=2$. The authors noticed a dichotomy in the existence range of $\lambda$ for $N=3$, relative to space dimensions $N \geqslant 4$. More precisely, for $N \geqslant 4$, there is a solution if and only if $\lambda \in\left(\frac{1}{4} N, \frac{1}{2} N\right)$. If $N=3$, there is a solution for $\lambda \in\left(1, \frac{3}{2}\right)$, there is no solution for $\lambda \leqslant \frac{3}{4}$ and $\lambda \geqslant \frac{3}{2}$. Complementing this result, it was proved in [1] that no radial solution exists for $\lambda \leqslant 1$. Here we present the situations where an analogous dichotomy happens, depending on the parameters $\alpha \geqslant 2$ and $\lambda$, thus extending the existence results of [11] for (1.1) and every $\alpha \geqslant 2$. We also show in detail a non-existence result similar to the one in [1], relative to radial solutions of (1.1). We address questions about symmetry breaking and multiplicity of solutions, where the least energy solutions of (1.1) are not radial, and there are at least two positive solutions.

Our main results are stated below in terms of the first eigenvalue,

$$
\lambda_{1}=\frac{1}{4} \alpha(N-2+\alpha),
$$

of the problem

$$
-\operatorname{div}(K(x) \nabla u)=\lambda K(x)|x|^{\alpha-2} u \quad \text { in } \mathbb{R}^{N} .
$$

We obtain solutions of (1.1) by minimizing the expression

$$
Q_{\lambda}(u)=\frac{\int_{\mathbb{R}^{N}} K(x)|\nabla u|^{2}-\lambda \int_{\mathbb{R}^{N}} K(x)|x|^{\alpha-2} u^{2}}{\left(\int_{\mathbb{R}^{N}} K(x)|u|^{2^{*}}\right)^{2 / 2^{*}}}
$$

over the space $H(\alpha)$ defined as the completion of the smooth functions with compact support $C_{\mathrm{c}}^{\infty}\left(\mathbb{R}^{N}\right)$ with respect to the norm

$$
\|u\|=\left(\int_{\mathbb{R}}^{N} K(x)|\nabla u|^{2}\right)^{1 / 2} .
$$

The infima of $Q_{\lambda}$ in $H(\alpha)$ are called ground states.

We shall prove the following results.

THEOREM 1.1 (strong weights). If $2<\alpha \leqslant N-2$, problem (1.1) has a solution if and only if $\lambda \in\left(\frac{1}{2} \lambda_{1}, \lambda_{1}\right)$. Moreover, in this situation the ground state is achieved.

Theorem 1.2 (very strong weights). If $N-2<\alpha$ and $\lambda \in\left(\frac{1}{4} \alpha^{2}, \lambda_{1}\right)$, problem (1.1) has a ground-state solution. Moreover, if $\lambda \leqslant \frac{1}{2} \lambda_{1}$ or $\lambda \geqslant \lambda_{1}$, then (1.1) has no solution.

The next results complement the ones above.

Theorem 1.3 (no radial solution). If $N-2<\alpha$ and $\lambda \leqslant \frac{1}{4} \alpha^{2}$, the problem (1.1) has no radial solution.

Theorem 1.4 (symmetry breaking). Let

$$
B^{2}=\frac{4^{2 / \alpha}}{\alpha^{2}} \sup _{0<y<\infty} \int_{0}^{y} \mathrm{e}^{s} s^{\mu-1} \mathrm{~d} s \int_{y}^{\infty} \mathrm{e}^{-s} s^{-\mu+(2 / \alpha)-1} \mathrm{~d} s,
$$

where $\mu=N / \alpha$. If

$$
\frac{1}{4 B^{2}}\left(1-\frac{\lambda}{\lambda_{1}}\right)>\frac{(N-1)(N-2)}{4}
$$


when either

$$
\frac{1}{2}<\frac{\lambda}{\lambda_{1}}<1 \quad \text { if } 2<\alpha \leqslant N-2
$$

or

$$
\frac{\alpha^{2}}{4 \lambda_{1}}<\frac{\lambda}{\lambda_{1}}<1 \quad \text { if } \alpha>N-2
$$

holds, then the ground-state solution of (1.1) exists and it is non-radial.

The motivation for studying such problems stems from a phenomenon noted in [6] which led to the notion of critical dimension (see [17]). Let $B_{1}(0)$ be the unit ball in $\mathbb{R}^{N}$, with $N \geqslant 3$. Let $\mu_{1}$ denote the first eigenvalue of the Laplacian with zero Dirichlet boundary conditions on $B_{1}(0)$. The following problem was studied in [6]:

$$
\left.\begin{array}{c}
-\Delta u=u^{2^{*}-1}+\lambda u, \quad u>0 \text { in } B_{1}(0), \\
u=0 \quad \text { on } \partial B_{1}(0) .
\end{array}\right\}
$$

The authors proved that, for $N \geqslant 4,(1.5)$ has a solution if and only if $\lambda \in\left(0, \mu_{1}\right)$, while, for $N=3$, problem (1.5) has a solution if and only if $\lambda \in\left(\frac{1}{4} \mu_{1}, \mu_{1}\right)$. The same phenomenon has been noted for problems with critical exponents on geodesic balls on the sphere $[2-4,7]$, and also for more general equations involving the $p$-Laplacian on the ball in $\mathbb{R}^{N}[9,10]$ or on the $N$-dimensional sphere [5]. A similar situation in $\mathbb{R}^{N}$ should be in some sense expected when the Dirichlet boundary conditions are replaced by a requirement that solutions have a fast decay at infinity. We impose this requirement by introducing the fast increasing weights $K(x)$. By the method developed in [6], we obtain solutions of (1.1) as critical points of $Q_{\lambda}$.

We note that for our existence results we consistently use radial test functions. We infer that, whenever a ground-state solution is achieved in $H(\alpha)$, the ground state in $H_{\mathrm{rad}}(\alpha)$ (radial functions in $\left.H(\alpha)\right)$ is also achieved. Indeed, this fact follows by repeating the original arguments in $H(\alpha)$ in the radial setting $H_{\mathrm{rad}}(\alpha)$. We do not know a priori whether the ground state in $H(\alpha)$ is radial. Theorem 1.4 guarantees cases when the ground state is not radial and, considering the least energy solution in $H_{\mathrm{rad}}(\alpha)$, we are thus led to the existence of at least two positive solutions of (1.1).

One is left with the following open question: are there situations when the ground state is achieved, but no radial solutions exist? According to our result above, if this happens, it has to be in the range $\alpha>N-2$ and $\frac{1}{2} \lambda_{1}<\lambda \leqslant \frac{1}{4} \alpha^{2}$.

The paper is structured as follows. After a brief section of preparatory results, we discuss in $\S 3$ the non-existence parts of theorems 1.1 and 1.2. Section 4 deals with the existence part of theorem 1.1. We prove theorem 1.2 in $\S 5$, and theorem 1.3 in $\S 6$. Finally, we present the symmetry-breaking argument that leads to theorem 1.4 in $\S 7$.

\section{Preliminaries}

Hereafter we write only $\int u$ to denote $\int_{\mathbb{R}^{N}} u(x) \mathrm{d} x$. For any $\alpha \geqslant 2$ we define $\theta(x)=\frac{1}{4}|x|^{\alpha}, K(x)=\exp (\theta(x))$, the Hilbert space $H(\alpha)$ as being the completion 
of $C_{\mathrm{c}}^{\infty}\left(\mathbb{R}^{N}\right)$ with respect to the norm

$$
\|u\|=\left(\int K(x)|\nabla u|^{2}\right)^{1 / 2}
$$

induced by the inner product

$$
(u, v)=\int K(x) \nabla u \cdot \nabla v .
$$

We define the weighted spaces

$$
L^{2}(\alpha)=\left\{u \text { measurable in } \mathbb{R}^{N}: \int K(x)|x|^{\alpha-2} u^{2}<\infty\right\}
$$

and

$$
L^{2^{*}}(\alpha)=\left\{u \text { measurable in } \mathbb{R}^{N}: \int K(x)|u|^{2^{*}}<\infty\right\}
$$

Proposition 2.1. The space $H(\alpha)$ is continuously embedded in $L^{2}(\alpha)$ and $L^{2^{*}}(\alpha)$.

Proof. For any $u \in C_{\mathrm{c}}^{\infty}\left(\mathbb{R}^{N}\right)$ we have

$$
\begin{aligned}
\int\left|\nabla\left(K(x)^{1 / 2} u\right)\right|^{2} & =\int\left|K(x)^{1 / 2} \nabla u+\nabla\left(K(x)^{1 / 2}\right) u\right|^{2} \\
& =\int K(x)|\nabla u|^{2}+\int \nabla\left(K(x)^{1 / 2} u^{2}\right) \cdot \nabla\left(K(x)^{1 / 2}\right) .
\end{aligned}
$$

Integrating by parts we get

$$
\begin{aligned}
\int\left|\nabla\left(K(x)^{1 / 2} u\right)\right|^{2} & =\int K(x)|\nabla u|^{2}-\int K(x)^{1 / 2} u^{2} \Delta\left(K(x)^{1 / 2}\right) \\
& =\int K(x)|\nabla u|^{2}-\frac{1}{2} \int K(x)\left(\Delta \theta(x)+\frac{1}{2}|\nabla \theta(x)|^{2}\right) u^{2} .
\end{aligned}
$$

From the Sobolev inequality we have

$$
\begin{aligned}
\int\left|\nabla\left(K(x)^{1 / 2} u\right)\right|^{2} & \geqslant S_{0}\left(\int\left(K(x)^{1 / 2}|u|\right)^{2^{*}}\right)^{2 / 2^{*}} \\
& \geqslant S_{0}\left(\int K(x)|u|^{2^{*}}\right)^{2 / 2^{*}}
\end{aligned}
$$

where $S_{0}>0$ denotes the best Sobolev constant. Hence,

$$
\int K(x)|\nabla u|^{2} \geqslant \frac{1}{2} \int K(x)\left(\Delta \theta(x)+\frac{1}{2}|\nabla \theta(x)|^{2}\right) u^{2}+S_{0}\left(\int K(x)|u|^{2^{*}}\right)^{2 / 2^{*}} .
$$

We therefore obtain

$$
\int K(x)|\nabla u|^{2} \geqslant \frac{1}{2} \int K(x)\left(\Delta \theta(x)+\frac{1}{2}|\nabla \theta(x)|^{2}\right) u^{2}
$$


and, since

$$
\begin{aligned}
\Delta \theta(x)+\frac{1}{2}|\nabla \theta(x)|^{2} & =|x|^{\alpha-2}\left(\frac{\alpha(N-2+\alpha)}{4}+\frac{\alpha^{2}}{32}|x|^{\alpha}\right) \\
& \geqslant \frac{\alpha(N-2+\alpha)}{4}|x|^{\alpha-2},
\end{aligned}
$$

we find from (2.1) that

$$
\int K(x)|\nabla u|^{2} \geqslant \frac{\alpha(N-2+\alpha)}{8} \int K(x)|x|^{\alpha-2} u^{2},
$$

which implies that $H(\alpha)$ embeds continuously in $L^{2}(\alpha)$, and

$$
\int K(x)|\nabla u|^{2} \geqslant S_{0}\left(\int K(x)|u|^{2^{*}}\right)^{2 / 2^{*}}
$$

which implies that $H(\alpha)$ embeds continuously into $L^{2^{*}}(\alpha)$.

Proposition 2.2. For any $\alpha \geqslant 2$ the space $H(\alpha)$ is compactly embedded in $L^{2}(\alpha)$.

Proof. In order to show that the embedding is compact, we proceed as in [11, proposition 1.1]. Let $\left(u_{n}\right) \subset H(\alpha)$ be such that

$$
u_{n} \rightarrow 0 \quad \text { weakly in } H(\alpha) \text { and }\left\|u_{n}\right\| \leqslant 1 .
$$

Given $\varepsilon>0$, we can use the definition of $\theta$ to obtain $R=R(\varepsilon)>0$ such that

$$
\Delta \theta(x)+\frac{1}{2}|\nabla \theta(x)|^{2}=|x|^{\alpha-2}\left(\frac{\alpha(N-2+\alpha)}{4}+\frac{\alpha^{2}}{32}|x|^{\alpha}\right) \geqslant \frac{2}{\varepsilon}|x|^{\alpha-2}
$$

for any $|x| \geqslant R$. Hence, by (2.2), we obtain

$$
\int_{\mathbb{R}^{N} \backslash B_{R}(0)} K(x)|x|^{\alpha-2} u_{n}^{2} \leqslant \varepsilon \int K(x)\left|\nabla u_{n}\right|^{2} \leqslant \varepsilon .
$$

On the other hand, arguments based on the Rellich-Kondrachov theorem imply that $u_{n} \rightarrow 0$ in $L^{2}\left(B_{R}(0)\right)$. Since $K(x)|x|^{\alpha-2} \in L^{\infty}\left(B_{R}(0)\right)$, it follows that

$$
\int_{B_{R}(0)} K(x)|x|^{\alpha-2} u_{n}^{2} \leqslant \varepsilon \text { for } n \geqslant n_{\varepsilon} .
$$

The above expression and (2.3) imply that $\int K(x)|x|^{\alpha-2} u_{n}^{2} \leqslant 2 \varepsilon$ for $n \geqslant n_{\varepsilon}$, i.e. $u_{n} \rightarrow 0$ strongly in $L^{2}(\alpha)$. The proposition is proved.

Proposition 2.2 and standard spectral theory for compact operators imply that the eigenvalue problem (1.3) has a sequence of eigenvalues

$$
0<\lambda_{1}<\lambda_{2} \leqslant \cdots \leqslant \lambda_{k} \leqslant \cdots
$$

such that $\lambda_{k} \rightarrow \infty$ as $k \rightarrow \infty$. Moreover, the first eigenvalue can be characterized as the infimum of the Rayleigh quotient

$$
\lambda_{1}=\inf _{u \in H^{1}(\alpha) \backslash\{0\}} \frac{\int|\nabla u|^{2} K(x)}{\int|u|^{2} K(x)|x|^{\alpha-2}} .
$$


We note that (1.3) is equivalent to

$$
-\Delta u-\frac{1}{4} \alpha(x \cdot \nabla u)|x|^{\alpha-2}=\lambda u|x|^{\alpha-2} .
$$

A direct calculation shows that $\varphi_{1}(x)=\exp \left(-\frac{1}{4}|x|^{\alpha}\right)>0$ is an eigenfunction associated with the first eigenvalue $\lambda_{1}=\frac{1}{4} \alpha(N-2+\alpha)$.

\section{Non-existence range}

In this section we deal with the non-existence parts of theorems 1.1 and 1.2. We use a Pohozaev identity to show that equation (1.1) has no non-trivial solution when $\lambda \leqslant \frac{1}{2} \lambda_{1}$. The result is quite flexible in the sense that it prevents not only positive solutions, but also sign-changing solutions in $H(\alpha)$ (see proposition 3.3). For the reader's convenience we give a proof of the following inequality.

Lemma 3.1 (Hardy inequality). Let $u \in C_{\mathrm{c}}^{\infty}\left(\mathbb{R}^{N}\right), a>-N$ and $R \geqslant 0$. Then

$$
\int_{\mathbb{R}^{N} \backslash B_{R}(0)}|u|^{2}|x|^{a} \leqslant \frac{4}{(N+a)^{2}} \int_{\mathbb{R}^{N} \backslash B_{R}(0)}(x \cdot \nabla u)^{2}|x|^{a} .
$$

Moreover, if $R=0$, the inequality is strict unless $u \equiv 0$.

Proof. Integrate the identity

$$
\operatorname{div}\left(u^{2}|x|^{a} x\right)=|x|^{a} x \cdot \nabla\left(u^{2}\right)+(N+a)|x|^{a} u^{2}
$$

on the domain $\mathbb{R}^{N} \backslash B_{R}(0)$ to get

$$
\begin{aligned}
0 & \geqslant-R^{a+1} \int_{\partial B_{R}(0)} u^{2} \mathrm{~d} \sigma \\
& =2 \int_{\mathbb{R}^{N} \backslash B_{R}(0)}|x|^{a} u x \cdot \nabla u+(N+a) \int_{\mathbb{R}^{N} \backslash B_{R}(0)}|x|^{a} u^{2} .
\end{aligned}
$$

The Cauchy-Schwarz inequality then gives

$$
2\left(\int_{\mathbb{R}^{N} \backslash B_{R}(0)}|x|^{a} u^{2}\right)^{1 / 2}\left(\int_{\mathbb{R}^{N} \backslash B_{R}(0)}|x|^{a}(x \cdot \nabla u)^{2}\right)^{1 / 2} \geqslant(N+a) \int_{\mathbb{R}^{N} \backslash B_{R}(0)}|x|^{a} u^{2},
$$

which is the desired inequality.

When $R=0$ the only way to have equality is if the functions $|u(x)|$ and $|x \cdot \nabla u(x)|$ are proportional. This can only happen when $u \equiv 0$.

REMARK 3.2. We note that when $a=\alpha-2$ lemma 3.1 holds for $u \in H(\alpha)$.

In the following we derive our non-existence result.

Proposition 3.3. Suppose that $u \in H(\alpha), \alpha \geqslant 2$, satisfies (1.2) and $\lambda \leqslant \frac{1}{2} \lambda_{1}$. Then $u \equiv 0$.

Proof. On multiplying (1.2) by $u$ and integrating by parts we obtain

$$
\int|\nabla u|^{2}-\frac{1}{8} \alpha \int|x|^{\alpha-2}\left(\nabla u^{2} \cdot x\right)=\int|u|^{2^{*}}+\lambda \int|x|^{\alpha-2} u^{2} .
$$


We now note that

$$
\int|x|^{\alpha-2}\left(\nabla u^{2} \cdot x\right)=-\int u^{2} \operatorname{div}\left(x|x|^{\alpha-2}\right)=-(N-2+\alpha) \int|x|^{\alpha-2} u^{2} ;
$$

hence, from (3.1) we obtain

$$
\int|\nabla u|^{2}+\frac{1}{8} \alpha(N-2+\alpha) \int|x|^{\alpha-2} u^{2}=\int|u|^{2^{*}}+\lambda \int|x|^{\alpha-2} u^{2} .
$$

On the other hand, we can multiply $(1.2)$ by $(x \cdot \nabla u)$ and integrate by parts once more to obtain

$$
\frac{N-2}{2} \int|\nabla u|^{2}+\frac{\alpha}{4} \int|x|^{\alpha-2}(x \cdot \nabla u)^{2}=\frac{N}{2^{*}} \int|u|^{2^{*}}+\frac{\lambda(N-2+\alpha)}{2} \int|x|^{\alpha-2} u^{2} .
$$

Suppose, by contradiction, that $u \not \equiv 0$. Combining the above expression with (3.2) we obtain

$$
\begin{aligned}
\left(\frac{1}{2} \alpha \lambda+\frac{1}{16} \alpha(N-2)(N-2+\alpha)\right) \int|x|^{\alpha-2} u^{2} & =\frac{1}{4} \alpha \int|x|^{\alpha-2}(x \cdot \nabla u)^{2} \\
& >\frac{1}{16} \alpha(N-2+\alpha)^{2} \int|x|^{\alpha-2} u^{2},
\end{aligned}
$$

where we have used the strict Hardy inequality of lemma 3.1. The above expression implies that

$$
\lambda>\frac{1}{8} \alpha(N-2+\alpha)=\frac{1}{2} \lambda_{1},
$$

which contradicts the hypothesis. Hence, $u \equiv 0$ and the proposition is proved.

It is also easy to see that (1.2) does not admit positive solutions when $\lambda \geqslant \lambda_{1}$. Indeed, since $\varphi_{1}$ satisfies (1.3), we assume that equation (1.1) has a positive solution, we multiply (1.1) by $\varphi_{1}$ and integrate by parts. We obtain

$$
\left(\lambda_{1}-\lambda\right) \int K(x) u \varphi_{1}=\int K(x) u^{2^{*}-1} \varphi_{1}
$$

which implies that $\lambda<\lambda_{1}$.

\section{Existence range}

In this section we prove the existence stated in theorem 1.1. We define

$$
S_{\lambda}=\inf _{u \in H(\alpha) \backslash\{0\}} Q_{\lambda}(u),
$$

and $S_{0}(K)$ in accordance with $\lambda=0$. For $\alpha \geqslant 2$ fixed and $\lambda \in \mathbb{R}$, we follow [6] by using the expression (1.4). We first note that $S_{0}(K)=S_{0}$, the best Sobolev constant. Indeed, from (2.1) we have $S_{0}(K) \geqslant S_{0}$. Using a smooth cut-off function $\varphi(x) \equiv 1$ in $B_{1}(0)$ and $\varphi(x) \equiv 0$ outside $B_{2}(0)$, and a sequence $u_{n}(x)=\varphi(x)\left(\varepsilon_{n}+\right.$ $\left.\left|x^{2}\right|\right)^{(2-N) / 2}$ with $\varepsilon_{n} \rightarrow 0$, we obtain $S_{0}(K) \leqslant \liminf _{n \rightarrow \infty} Q_{0}\left(u_{n}\right)=S_{0}$.

The existence result will be proved by checking that, under the hypotheses of theorem 1.1, the number $S_{\lambda}$ is achieved. This is exactly the content of the next two results. 
Lemma 4.1. If $0<S_{\lambda}(K)<S_{0}(K)$, then $S_{\lambda}(K)$ is achieved.

Proof. It suffices to argue as in [11, lemma 4.11].

Proposition 4.2. If $2 \leqslant \alpha \leqslant N-2$, for any $\lambda \in\left(\frac{1}{2} \lambda_{1}, \lambda_{1}\right)$ we have $0<S_{\lambda}(K)<$ $S_{0}(K)$.

Proof. Let $\varphi \in C^{\infty}\left(\mathbb{R}^{N},[0,1]\right)$ be such that $\varphi \equiv 1$ on $B_{1}(0)$ and $\varphi \equiv 0$ outside $B_{2}(0)$. Given $a \geqslant 2$ and $b \geqslant 0$, we can easily check that

$$
\int|x|^{a}\left(1+|x|^{2}\right)^{b-N}<\infty \quad \text { provided that } N>a+2 b .
$$

In this case, we can compute

$$
\begin{aligned}
\int \frac{\varphi^{2}|x|^{a}}{\left(\varepsilon+|x|^{2}\right)^{N-b}} & =\int \frac{|x|^{a}}{\left(\varepsilon+|x|^{2}\right)^{N-b}}+\int \frac{\left(\varphi^{2}-1\right)|x|^{a}}{\left(\varepsilon+|x|^{2}\right)^{N-b}} \\
& =O(1)+\int \frac{\varepsilon^{a / 2}|x / \sqrt{\varepsilon}|^{a}}{\varepsilon^{N-b}\left(1+|x / \sqrt{\varepsilon}|^{2}\right)^{N-b}} \\
& =O(1)+\varepsilon^{b+a / 2-N / 2} \int \frac{|x|^{a}}{\left(1+|x|^{2}\right)^{N-b}},
\end{aligned}
$$

as $\varepsilon \rightarrow 0^{+}$.

For $\varepsilon>0$, let us define

$$
u_{\varepsilon}=K^{-1 / 2} \varphi v_{\varepsilon},
$$

where $v_{\varepsilon}$ is the 'instanton' given by

$$
v_{\varepsilon}(x)=\left(\frac{1}{\varepsilon+|x|^{2}}\right)^{(N-2) / 2} .
$$

Our aim is to check that, for small values of $\varepsilon$, we have $Q_{\lambda}\left(u_{\varepsilon}\right)<S$. In order to do this, we first compute

$$
\begin{aligned}
\int\left|\nabla u_{\varepsilon}\right|^{2} K=\int \varphi^{2}\left(\left|\nabla v_{\varepsilon}\right|^{2}-\right. & \left.\frac{\alpha}{4} v_{\varepsilon}\left(x \cdot \nabla v_{\varepsilon}\right)|x|^{\alpha-2}+\frac{\alpha^{2}}{4 \cdot 16} v_{\varepsilon}^{2}|x|^{2(\alpha-1)}\right) \\
& +\int v_{\varepsilon}^{2}|\nabla \varphi|^{2}+2 \int \varphi v_{\varepsilon} \nabla \varphi \cdot\left(\nabla v_{\varepsilon}-\frac{\alpha}{8} v_{\varepsilon}|x|^{\alpha-2} x\right) .
\end{aligned}
$$

It is easy to check that the two last terms of the right-hand side are bounded as $\varepsilon \rightarrow 0$. Thus, by using the definition of $v_{\varepsilon}$, we get

$$
\begin{aligned}
\int\left|\nabla u_{\varepsilon}\right|^{2} K= & (2-N)^{2} \int \frac{\varphi^{2}|x|^{2}}{\left(\varepsilon+|x|^{2}\right)^{N}}+\frac{\alpha(N-2)}{4} \int \frac{\varphi^{2}|x|^{\alpha}}{\left(\varepsilon+|x|^{2}\right)^{N-1}} \\
& +\frac{\alpha^{2}}{4 \cdot 16} \int \frac{\varphi^{2}|x|^{2(\alpha-1)}}{\left(\varepsilon+|x|^{2}\right)^{N-2}}+O(1) \\
= & I_{1}+I_{2}+I_{3}+O(1) .
\end{aligned}
$$


By using the estimate in (4.1) we obtain

$$
\left.\begin{array}{ll}
I_{1}=\varepsilon^{1-N / 2} A_{1}+O(1) & (\text { for } N>2), \\
I_{2}=\varepsilon^{\alpha / 2+(1-N / 2)} A_{2}+O(1) & (\text { for } N>\alpha+2), \\
I_{3}=\varepsilon^{\alpha+(1-N / 2)} A_{3}+O(1) & (\text { for } N>2 \alpha+2),
\end{array}\right\}
$$

with

$$
\begin{array}{ll}
\left.A_{1}:=(N-2)^{2} \int \frac{|x|^{2}}{\left(1+|x|^{2}\right)^{N}} \quad \text { (for } N>2\right), \\
A_{2}:=\frac{\alpha(N-2)}{4} \int \frac{|x|^{\alpha}}{\left(1+|x|^{2}\right)^{N-1}} & (\text { for } N>\alpha+2), \\
\left.A_{3}:=\frac{\alpha^{2}}{4 \cdot 16} \int \frac{|x|^{2(\alpha-1)}}{\left(1+|x|^{2}\right)^{N-2}} \quad \text { (for } N>2 \alpha+2\right) .
\end{array}
$$

In the same way, we have

$$
\begin{aligned}
\lambda \int\left|u_{\varepsilon}\right|^{2} K|x|^{\alpha-2} & =\lambda \int \frac{\varphi^{2}|x|^{\alpha-2}}{\left(\varepsilon+|x|^{2}\right)^{N-2}} \\
& =\varepsilon^{\alpha / 2+(1-N / 2)} \lambda A_{4}+O(1) \quad(\text { for } N>\alpha+2)
\end{aligned}
$$

where

$$
A_{4}:=\int \frac{|x|^{\alpha-2}}{\left(1+|x|^{2}\right)^{N-2}} \quad(\text { for } N>\alpha+2) .
$$

Arguing as in (4.1), we can compute

$$
\int\left|u_{\varepsilon}\right|^{2^{*}} K=\int \frac{\varphi^{2^{*}} K^{2 /(2-N)}}{\left(\varepsilon+|x|^{2}\right)^{N}}=\varepsilon^{-N / 2} A_{0}+O(1) \quad(\text { for } N>2),
$$

where

$$
A_{0}:=\int \frac{1}{\left(1+|x|^{2}\right)^{N}} \quad(\text { for } N>2)
$$

Hence, we get

$$
\begin{aligned}
\left(\int\left|u_{\varepsilon}\right|^{2^{*}} K\right)^{2 / 2^{*}} & =\left(\varepsilon^{-N / 2} A_{0}+O(1)\right)^{2 / 2^{*}} \\
& =\left(\varepsilon^{-N / 2} A_{0}\right)^{2 / 2^{*}}+\frac{N-2}{N}\left(\varepsilon^{-N / 2} A_{0}+O(1)\right)^{-2 / N} O(1),
\end{aligned}
$$

and therefore

$$
\left(\int\left|u_{\varepsilon}\right|^{2^{*}} K\right)^{2 / 2^{*}}=\varepsilon^{1-N / 2} A_{0}^{(N-2) / N}+O(\varepsilon) \quad(\text { for } N>2) .
$$

We now consider several distinct cases depending on the values of $N$ and $\alpha$. 
CASE $1(N>2 \alpha+2)$. In this case, all the equalities in (4.2)-(4.4) hold and we have

$$
\begin{aligned}
Q_{\lambda}\left(u_{\varepsilon}\right) & =\frac{\varepsilon^{1-N / 2}\left(A_{1}+\varepsilon^{\alpha / 2}\left(A_{2}-\lambda A_{4}\right)+\varepsilon^{\alpha} A_{3}\right)+O(1)}{\varepsilon^{1-N / 2} A_{0}^{(N-2) / N}+O(\varepsilon)} \\
& =A_{0}^{-1+2 / N}\left(A_{1}+\varepsilon^{\alpha / 2}\left(A_{2}-\lambda A_{4}\right)+\varepsilon^{\alpha} A_{3}+O\left(\varepsilon^{N / 2-1}\right)\right) \\
& <A_{0}^{-1+2 / N} A_{1},
\end{aligned}
$$

for $\varepsilon>0$ small enough, provided that $\lambda>A_{2} / A_{4}$. However, it is well known [6] that $A_{0}^{-1+2 / N} A_{1}=S_{0}=S_{0}(K)$ and therefore it remains to check that

$$
\frac{A_{2}}{A_{4}}=\frac{1}{8} \alpha(N+\alpha-2) \quad(\text { for } N>\alpha+2) .
$$

In order to do this, we denote by $\omega_{N-1}$ the area of the sphere $\mathbb{S}^{N-1} \subset \mathbb{R}^{N}$ and integrate by parts to get

$$
\begin{aligned}
A_{4} & =\int \frac{|x|^{\alpha-2}}{\left(1+|x|^{2}\right)^{N-2}} \\
& =\omega_{N-1} \int_{0}^{\infty} r^{(N+\alpha-2)-1} \frac{1}{\left(1+r^{2}\right)^{N-2}} \mathrm{~d} r \\
& =\frac{2(N-2)}{(N+\alpha-2)} \omega_{N-1} \int_{0}^{\infty} \frac{r^{N+\alpha-1}}{\left(1+r^{2}\right)^{N-1}} \mathrm{~d} r \\
& =\frac{8}{\alpha(N+\alpha-2)} A_{2} .
\end{aligned}
$$

Thus, (4.5) holds and the proposition is proved in the case when $N>2 \alpha+2$.

CASE $2(N=2 \alpha+2)$. In this case, we need to perform another estimate for $I_{3}$. Thus, we first note that

$$
\int_{B_{1}(0)} \frac{|x|^{2(\alpha-1)}}{\left(\varepsilon+|x|^{2}\right)^{2 \alpha}} \leqslant I_{3} \leqslant \int_{B_{2}(0)} \frac{|x|^{2(\alpha-1)}}{\left(\varepsilon+|x|^{2}\right)^{2 \alpha}} .
$$

On the other hand, for any $R>0$, it holds that

$$
\begin{aligned}
\int_{B_{R}(0)} \frac{|x|^{2(\alpha-1)}}{\left(\varepsilon+|x|^{2}\right)^{2 \alpha}} & =\frac{1}{2} \omega_{N-1} \int_{0}^{R} \frac{r^{2(\alpha-1)} r^{2 \alpha}(2 r)}{\left(\varepsilon+r^{2}\right)^{2 \alpha}} \mathrm{d} r \\
& =\frac{1}{2} \omega_{N-1} \int_{\varepsilon}^{\varepsilon+R^{2}} \frac{(s-\varepsilon)^{2 \alpha-1}}{s^{2 \alpha}} \mathrm{d} s \\
& =\frac{1}{2} \omega_{N-1}\left(\int_{\varepsilon}^{\varepsilon+R^{2}} \frac{1}{s} \mathrm{~d} s+\int_{\varepsilon}^{\varepsilon+R^{2}} \sum_{i=1}^{2 \alpha-1} C_{i} \frac{s^{(2 \alpha-1)-i}}{s^{2 \alpha}} \varepsilon^{i} \mathrm{~d} s\right) \\
& =\frac{1}{2} \omega_{N-1} \log \left|\varepsilon+R^{2}\right|-\frac{1}{2} \omega_{N-1} \log |\varepsilon|+O(1) \\
& =\frac{1}{2} \omega_{N-1}|\log \varepsilon|+O(1) .
\end{aligned}
$$


Hence, as in case 1 , we get

$$
\begin{aligned}
\int\left|\nabla u_{\varepsilon}\right|^{2} K & =\varepsilon^{1-N / 2}\left(A_{1}+\varepsilon^{\alpha / 2} A_{2}+\frac{1}{2} \varepsilon^{-1+N / 2} \omega_{N-1}|\log \varepsilon|\right)+O(1) \\
& =\varepsilon^{1-N / 2}\left(A_{1}+\varepsilon^{\alpha / 2} A_{2}+\frac{1}{2} \varepsilon^{\alpha} \omega_{N-1}|\log \varepsilon|\right)+O(1)
\end{aligned}
$$

and therefore

$$
Q_{\lambda}\left(u_{\varepsilon}\right)=A_{0}^{-1+2 / N}\left(A_{1}+\varepsilon^{\alpha / 2}\left(A_{2}-\lambda A_{4}\right)+\frac{1}{2} \varepsilon^{\alpha} \omega_{N-1}|\log \varepsilon|+O\left(\varepsilon^{N / 2-1}\right)\right)<S_{0}
$$

for $\varepsilon$ sufficiently small.

CASE $3(\alpha+2<N<2 \alpha+2)$. In this case, since $N<2 \alpha+2$, we can estimate $I_{3}$ as follows:

$$
I_{3}=\frac{\alpha^{2}}{4 \cdot 16} \int_{B_{2}(0)} \frac{\varphi^{2}|x|^{2(\alpha-1)}}{\left(\varepsilon+|x|^{2}\right)^{N-2}} \leqslant \int_{B_{2}(0)} \frac{|x|^{2(\alpha-1)}}{|x|^{2(N-2)}}=O(1)
$$

and therefore

$$
Q_{\lambda}\left(u_{\varepsilon}\right)=A_{0}^{-1+2 / N}\left(A_{1}+\varepsilon^{\alpha / 2}\left(A_{2}-\lambda A_{4}\right)+O\left(\varepsilon^{N / 2-1}\right)\right)<S_{0}
$$

for $\varepsilon$ small enough.

CASE $4(N=\alpha+2)$. In this case $I_{3}=O(1)$. However, (4.3) does not hold and we also need to estimate $I_{2}$. But this can be made as in case 2 , and we can check that

$$
I_{2}=\frac{1}{2} \omega_{N-1} \frac{1}{4} \alpha(N-2)|\log \varepsilon|+O(1)
$$

and

$$
\lambda \int\left|u_{\varepsilon}\right|^{2} K|x|^{\alpha-2}=\frac{1}{2} \omega_{N-1}|\log \varepsilon|+O(1) .
$$

Hence,

$$
Q_{\lambda}\left(u_{\varepsilon}\right)=A_{0}^{-1+2 / N}\left(A_{1}+\frac{1}{2} \omega_{N-1}\left(\frac{1}{4} \alpha(N-2)-\lambda\right)+O\left(\varepsilon^{N / 2-1}\right)\right)<S_{0},
$$

provided $\varepsilon$ is small and

$$
\lambda>\frac{1}{4} \alpha(N-2)=\frac{1}{4} \alpha^{2}=\frac{1}{8} \alpha(N+\alpha-2) .
$$

This concludes the proof of the proposition.

\section{Existence when $N<\alpha+2$ and $\frac{1}{4} \alpha^{2}<\lambda<\frac{1}{4} \alpha(N-2+\alpha)$}

In this section we prove the existence statement of theorem 1.2. For this we work with test functions using the solutions

$$
U(x)=c_{N}\left(\frac{\varepsilon}{\varepsilon^{2}+|x|^{2}}\right)^{(N-2) / 2}
$$


of $-\Delta U=U^{2^{*}-1}$. For convenience, define $\varphi(x)=K^{-1 / 2}(x)=\exp \left(-\frac{1}{8}|x|^{\alpha}\right)$ and let $u=\varphi^{2} U$. We calculate

$$
\begin{aligned}
\int K|\nabla u|^{2} & =\int \varphi^{-2}\left|\nabla\left(\varphi^{2} U\right)\right|^{2} \\
& =\int|2 U \nabla \varphi+\varphi \nabla U|^{2} \\
& =\int 4 U^{2}|\nabla \varphi|^{2}+2 \varphi U(\nabla \varphi \cdot \nabla U)+\operatorname{div}\left(\varphi^{2} U \nabla U\right)-\varphi^{2} U \Delta U \\
& =\int 4 U^{2}|\nabla \varphi|^{2}+\frac{1}{2}\left(\nabla \varphi^{2} \cdot \nabla U^{2}\right)+\varphi^{2} U^{2^{*}}+\operatorname{div}\left(\varphi^{2} U \nabla U\right) \\
& =\int 4 U^{2}|\nabla \varphi|^{2}-\frac{1}{2} U^{2} \Delta \varphi^{2}+\varphi^{2} U^{2^{*}}+\operatorname{div}\left(\varphi^{2} U \nabla U+\frac{1}{2} U^{2} \nabla \varphi^{2}\right) .
\end{aligned}
$$

Since the divergences integrate to zero, we get

$$
\int K|\nabla u|^{2}=\int \varphi^{2} U^{2^{*}}+\int U^{2}\left(4|\nabla \varphi|^{2}-\frac{1}{2} \Delta \varphi^{2}\right) .
$$

Using the explicit form of $\varphi$, a direct calculation yields

$$
4|\nabla \varphi|^{2}-\frac{1}{2} \Delta \varphi^{2}=\frac{1}{8} \alpha(N+\alpha-2)|x|^{\alpha-2} \varphi^{2}+\frac{1}{32} \alpha^{2}|x|^{2 \alpha-2} \varphi^{2}
$$

so we can write

$$
\int U^{2}\left(4|\nabla \varphi|^{2}-\frac{1}{2} \Delta \varphi^{2}\right)=\int U^{2}|x|^{\alpha-2} \Psi(x),
$$

where $\Psi(x)=\frac{1}{8} \alpha(N+\alpha-2) \varphi^{2}+\frac{1}{32} \alpha^{2}|x|^{\alpha} \varphi^{2}$.

We now show that

$$
\int U^{2}|x|^{\alpha-2} \Psi(x)=c_{N}^{2} \varepsilon^{N-2} \int|x|^{\alpha+2-2 N} \Psi(x)+o\left(\varepsilon^{N-2}\right) ;
$$

therefore,

$$
\begin{aligned}
\int K|\nabla u|^{2}=\int \varphi^{2} U^{2^{*}}+c_{N}^{2} \varepsilon^{N-2} & \frac{1}{8} \alpha(N+\alpha-2) \int \frac{|x|^{\alpha-2}}{|x|^{2(N-2)}} \varphi^{2} \\
& +c_{N}^{2} \varepsilon^{N-2} \frac{1}{32} \alpha^{2} \int \frac{|x|^{2 \alpha-2}}{|x|^{2(N-2)}} \varphi^{2}+o\left(\varepsilon^{N-2}\right) .
\end{aligned}
$$

Indeed,

$$
\begin{aligned}
\int|x|^{\alpha+2-2 N} \Psi(x)- & \int \frac{|x|^{\alpha-2}}{\left(|x|^{2}+\varepsilon^{2}\right)^{N-2}} \Psi(x) \\
& =\int \frac{|x|^{\alpha+2-2 N}\left(\left(|x|^{2}+\varepsilon^{2}\right)^{N-2}-|x|^{2(N-2)}\right)}{\left(|x|^{2}+\varepsilon^{2}\right)^{N-2}} \Psi(x)
\end{aligned}
$$




$$
\begin{aligned}
& =\sum_{k=1}^{N-2}\left(\begin{array}{c}
N-2 \\
k
\end{array}\right) \int \frac{|x|^{\alpha+2-2 N}|x|^{2(N-2-k)} \varepsilon^{2 k}}{\left(|x|^{2}+\varepsilon^{2}\right)^{N-2}} \Psi(x) \\
& \leqslant \sum_{k=1}^{N-2}\left(\begin{array}{c}
N-2 \\
k
\end{array}\right) \int \frac{|x|^{\alpha+2-2 N} \varepsilon^{2 k}}{\left(|x|^{2}+\varepsilon^{2}\right)^{k}} \Psi(x) .
\end{aligned}
$$

Each of the integrals

$$
\int \frac{|x|^{\alpha+2-2 N} \varepsilon^{2 k}}{\left(|x|^{2}+\varepsilon^{2}\right)^{k}} \Psi(x)
$$

is estimated as follows. Pick

$$
0<\mu<\min \left\{\frac{\alpha}{N-2}-1,2\right\}, \quad p=\frac{2}{\mu}, \quad q=\frac{2}{2-\mu} .
$$

We use Young's inequality,

$$
\frac{a^{p}}{p}+\frac{b^{q}}{q} \geqslant a b, \quad \text { with } a=\left(p|x|^{2}\right)^{1 / p} \text { and } b=\left(q \varepsilon^{2}\right)^{1 / q} .
$$

Then

$$
|x|^{2}+\varepsilon^{2} \geqslant \frac{2}{\mu^{\mu / 2}(2-\mu)^{(2-\mu) / 2}}|x|^{\mu} \varepsilon^{2-\mu}
$$

hence,

$$
\int \frac{|x|^{\alpha+2-2 N} \varepsilon^{2 k}}{\left(|x|^{2}+\varepsilon^{2}\right)^{k}} \Psi(x) \leqslant \frac{\mu^{k \mu / 2}(2-\mu)^{k(2-\mu) / 2}}{2^{k}} \int|x|^{\alpha+2-2 N-k \mu} \varepsilon^{k \mu} \Psi(x) .
$$

From $\alpha+2-2 N-k \mu>-N$ we find that every integral on the right-hand side is convergent. Hence,

$$
\int \frac{|x|^{\alpha+2-2 N} \varepsilon^{2 k}}{\left(|x|^{2}+\varepsilon^{2}\right)^{k}} \Psi(x) \leqslant C(k, \mu) \varepsilon^{k \mu}
$$

and this proves (5.1).

Returning to (5.2), we use

$$
0=\int \operatorname{div}\left(\frac{|x|^{\alpha-2} x}{|x|^{2(N-2)}} \varphi^{2}\right)=(\alpha-N+2) \int \frac{|x|^{\alpha-2}}{|x|^{2(N-2)}} \varphi^{2}-\frac{1}{4} \alpha \int \frac{|x|^{2 \alpha-2}}{|x|^{2(N-2)}} \varphi^{2}
$$

from which we may infer that

$$
\frac{1}{32} \alpha^{2} \int \frac{|x|^{2 \alpha-2}}{|x|^{2(N-2)}} \varphi^{2}=\frac{1}{8} \alpha(\alpha-N+2) \int \frac{|x|^{\alpha-2}}{|x|^{2(N-2)}} \varphi^{2}
$$

Therefore,

$$
\int K|\nabla u|^{2}=\int \varphi^{2} U^{2^{*}}+c_{N}^{2} \varepsilon^{N-2} \frac{1}{4} \alpha^{2} \int \frac{|x|^{\alpha-2}}{|x|^{2(N-2)}} \varphi^{2}+o\left(\varepsilon^{N-2}\right) .
$$

The same estimates used for (5.1) give

$$
\lambda \int K|x|^{\alpha-2} u^{2}=\lambda c_{N}^{2} \varepsilon^{N-2} \int \frac{|x|^{\alpha-2}}{|x|^{2(N-2)}} \varphi^{2}+o\left(\varepsilon^{N-2}\right)
$$


and so

$$
\begin{aligned}
\int K|\nabla u|^{2}-\lambda & \int K|x|^{\alpha-2} u^{2} \\
& =\int \varphi^{2} U^{2^{*}}+c_{N}^{2} \varepsilon^{N-2}\left(\frac{1}{4} \alpha^{2}-\lambda\right) \int \frac{|x|^{\alpha-2}}{|x|^{2(N-2)}} \varphi^{2}+o\left(\varepsilon^{N-2}\right) .
\end{aligned}
$$

Also

$$
\int K u^{2^{*}}=\int \varphi^{2(N+2) /(N-2)} U^{2^{*}} .
$$

We show that, for $p>0$, we can write estimates of the type

$$
\int \varphi^{p} U^{2^{*}}=\int U^{2^{*}}+o\left(\varepsilon^{N-2}\right) .
$$

This is because

$$
\int \varphi^{p} U^{2^{*}}=\int U^{2^{*}}+\int\left(1-\varphi^{p}\right) c_{N}^{2^{*}} \frac{\varepsilon^{N}}{\left(|x|^{2}+\varepsilon^{2}\right)^{N}} .
$$

Using Young's inequality (5.3) again, with

$$
\min \left\{\frac{\alpha+N}{N}, 2\right\}>\mu>2 \frac{N-1}{N},
$$

we obtain

$$
\int\left(1-\varphi^{p}\right) c_{N}^{2^{*}} \frac{\varepsilon^{N}}{\left(|x|^{2}+\varepsilon^{2}\right)^{N}} \leqslant c_{N}^{2^{*}} \frac{\mu^{N \mu / 2}(2-\mu)^{N(2-\mu) / 2}}{2^{N}} \int\left(1-\varphi^{p}\right) \frac{\varepsilon^{N(\mu-1)}}{|x|^{N \mu}},
$$

which proves (5.4) because the exponent of $\varepsilon$ is

$$
N(\mu-1)>N-2
$$

and the integral is convergent since, for $|x|$ small,

$$
1-\varphi^{p}(x) \leqslant \frac{1}{4} p|x|^{\alpha} \quad \text { and } \quad \alpha+N-1-N \mu>-1 .
$$

Then, the definition of $Q_{\lambda}(u)$ gives

$$
Q_{\lambda}(u)=\frac{\int U^{2^{*}}+c_{N}^{2} \varepsilon^{N-2}\left(\frac{1}{4} \alpha^{2}-\lambda\right) \int\left(|x|^{\alpha-2} /|x|^{2(N-2)}\right) \varphi^{2}+o\left(\varepsilon^{N-2}\right)}{\left(\int U^{2^{*}}+o\left(\varepsilon^{N-2}\right)\right)^{2 / 2^{*}}}<S_{0}
$$

for small $\varepsilon>0$, provided that $\lambda>\frac{1}{4} \alpha^{2}$.

\section{The radial case for $N<\alpha+2$ and $0<\lambda \leqslant \frac{1}{4} \alpha^{2}$}

In this section we prove theorem 1.3, so we show non-existence of positive radial solutions in the case $N<\alpha+2$ and $0<\lambda \leqslant \frac{1}{4} \alpha^{2}$. The argument is more general than that in [1] for the case $\alpha=2$. We use the method in [8] to obtain a Pohozaevtype identity. 
Positive radial solutions $u=u(r)$ of equation (1.2) satisfy

$$
-u_{r r}-\left(\frac{N-1}{r}+\frac{\alpha r^{\alpha-1}}{4}\right) u_{r}=\lambda r^{\alpha-2} u+u^{2^{*}-1} .
$$

We set

$$
h(r)=\frac{N-1}{r}+\frac{\alpha r^{\alpha-1}}{4} \text { and } H(r)=r^{N-1} \mathrm{e}^{r^{\alpha} / 4} .
$$

Note that $H_{r}=h H$. After multiplication by $H,(6.1)$ can be written as

$$
-\left(H u_{r}\right)_{r}=\lambda r^{\alpha-2} H u+H u^{2^{*}-1} .
$$

Let $\xi$ and $\zeta$ be solutions of the linearized (6.2) about $u \equiv 0$, i.e. solutions of

$$
-\left(H \xi_{r}\right)_{r}=\lambda r^{\alpha-2} H \xi
$$

We define the Wronskian of $u$ and $\xi$ as

$$
W[u, \xi](r)=H(r)\left(u(r) \xi_{r}(r)-u_{r}(r) \xi(r)\right) .
$$

After multiplying equation (6.2) by $\xi$ and equation (6.3) by $-u$, we add the two equalities to obtain

$$
\frac{\mathrm{d}}{\mathrm{d} r} W[u, \xi]=H u^{2^{*}-1} \xi
$$

Similarly, by using the solution $\zeta$ instead of $\xi$ we obtain

$$
\frac{\mathrm{d}}{\mathrm{d} r} W[u, \zeta]=H u^{2^{*}-1} \zeta .
$$

We multiply equality $(6.4)$ by $W[u, \zeta]$ and equality $(6.5)$ by $W[u, \xi]$ and add the results. We therefore obtain

$$
\frac{\mathrm{d}}{\mathrm{d} r}(W[u, \xi] W[u, \zeta])=H u^{2^{*}-1}(\xi W[u, \zeta]+\zeta W[u, \xi]) .
$$

A straightforward calculation yields

$$
\frac{\mathrm{d}}{\mathrm{d} r}\left(W[u, \xi] W[u, \zeta]+\frac{2}{2^{*}} H^{2} u^{2^{*}} \xi \zeta\right)=\frac{2+2^{*}}{2^{*}} H^{\left(2 \cdot 2^{*}\right) /\left(2+2^{*}\right)} u^{2^{*}}\left(H^{4 /\left(2+2^{*}\right)} \xi \zeta\right)_{r}
$$

We now show how to pick suitable $\xi$ and $\zeta$, positive solutions of (6.3), so that if $N<\alpha+2$ and $0<\lambda<\frac{1}{4} \alpha^{2}$, the right-hand side of (6.6) is negative for all $r>0$. That is

$$
\left(H^{4 /\left(2+2^{*}\right)} \xi \zeta\right)_{r}<0
$$

which-because $\xi$ and $\zeta$ will be positive - is equivalent to

$$
\frac{N-2}{r}+\frac{N-2}{N-1} \frac{\alpha r^{\alpha-1}}{4}+\frac{\xi_{r}}{\xi}+\frac{\zeta_{r}}{\zeta}<0
$$

Making the change of variables

$$
s=\frac{1}{4} r^{\alpha}, \quad \xi(r)=\mathrm{e}^{-s} f(s), \quad \zeta(r)=\mathrm{e}^{-s} g(s),
$$


equation (6.3) for $\xi$ and $\zeta$ transforms into the confluent hypergeometric equation, also known as Kummer's equation, for $f$ and $g$ :

$$
s f_{s s}+(b-s) f_{s}-a f=0,
$$

where $b=(N-2+\alpha) / \alpha \in(1,2)$ and $a=(N-2+\alpha) / \alpha-\left(4 \lambda / \alpha^{2}\right) \in(0, b)$. Let $\Phi(a, b ; s)$ and $\Psi(a, b ; s)$ be the confluent hypergeometric function and confluent hypergeometric function of the second kind, respectively (see [15, $\S \S 9.9-9.12]$ ).

We define

$$
f(s)=\frac{\Gamma(a) \Gamma(b-a)}{\Gamma(b)} \Phi(a, b ; s) \quad \text { and } \quad g(s)=\Gamma(a) \Psi(a, b ; s)
$$

so that $f$ and $g$ solve (6.8) and have the integral representations

$$
f(s)=\mathrm{e}^{s} \int_{0}^{1} \mathrm{e}^{-s t} t^{b-a-1}(1-t)^{a-1} \mathrm{~d} t
$$

and

$$
g(s)=\mathrm{e}^{s} \int_{1}^{\infty} \mathrm{e}^{-s t} t^{b-a-1}(t-1)^{a-1} \mathrm{~d} t .
$$

Using the differentiation formula (9.10.12) and the recurrence relations (9.10.14) and $(9.10 .13)$ in $[15]$ we have

$$
\begin{aligned}
\frac{\mathrm{d}}{\mathrm{d} s} \Psi(a, b ; s) & =-a \Psi(a+1, b+1 ; s), \\
\Psi(a+1, b+1 ; s) & =\frac{b-a-1}{s} \Psi(a+1, b ; s)+\frac{1}{s} \Psi(a, b ; s), \\
\Psi(a+1, b ; s) & =\frac{1}{a} \Psi(a, b ; s)-\frac{1}{a} \Psi(a, b-1 ; s) .
\end{aligned}
$$

Combining the three equalities above we get

$$
\frac{\mathrm{d}}{\mathrm{d} s} \Psi(a, b ; s)=-\frac{b-1}{s} \Psi(a, b ; s)+\frac{b-a-1}{s} \Psi(a, b-1 ; s) .
$$

After multiplication by $\Gamma(a)$ this becomes

$$
\frac{\mathrm{d}}{\mathrm{d} s} g+\frac{b-1}{s} g=\frac{b-a-1}{s} \mathrm{e}^{s} \int_{1}^{\infty} \mathrm{e}^{-s t} t^{b-a-2}(t-1)^{a-1} \mathrm{~d} t .
$$

Changing the variables back to $r$ and $\zeta$, we obtain

$$
\zeta_{r}+\left(\frac{\alpha r^{\alpha-1}}{4}+\frac{(b-1) \alpha}{r}\right) \zeta=\frac{\alpha(b-a-1)}{r} \int_{1}^{\infty} \mathrm{e}^{-r^{\alpha} t / 4} t^{b-a-2}(t-1)^{a-1} \mathrm{~d} t
$$

When

$$
b-a-1<0, \quad \text { i.e. } \lambda<\frac{1}{4} \alpha^{2},
$$

we find that the left-hand side of (6.9) is negative, and, hence,

$$
\frac{\zeta_{r}}{\zeta}<-\frac{\alpha r^{\alpha-1}}{4}-\frac{N-2}{r}
$$

Since $\xi_{r} / \xi$ is also negative, inequality (6.7) follows from the inequality above. 
Assuming that (6.2) has a positive solution, we get a contradiction, as follows: let

$$
\mathcal{H}=W[u, \xi] W[u, \zeta]+\frac{2}{2^{*}} H^{2} u^{2^{*}} \xi \zeta
$$

be the expression differentiated on the left-hand side of (6.6). Then

$$
\mathcal{H}=H^{2}\left(u_{r}^{2} \xi \zeta-u_{r} u(\xi \zeta)_{r}+u^{2} \xi_{r} \zeta_{r}+\frac{2}{2^{*}} u^{2^{*}} \xi \zeta\right) .
$$

Since we have

$$
\xi(r)=O(1) \quad \text { and } \quad \zeta(r)=O\left(r^{\alpha(1-b)}\right)=O\left(r^{-(N-2)}\right)
$$

near $r=0$, we obtain

$$
\lim _{r \rightarrow 0^{+}} \mathcal{H}(r)=0 .
$$

On the other hand, for large $r$ we have

$$
\xi(r)=O\left(r^{-\alpha(b-a)}\right)=O\left(r^{-4 \lambda / \alpha}\right)
$$

and

$$
\zeta(r)=O\left(\mathrm{e}^{-r^{\alpha} / 4} r^{-\alpha a}\right)=O\left(\mathrm{e}^{-r^{\alpha} / 4} r^{-(N-2+\alpha-(4 \lambda / \alpha))}\right)
$$

These estimates, together with the requirements (which follow from $u \in H(\alpha)$ ) that

$$
\int_{0}^{\infty} H u_{r}^{2}<\infty, \quad \int_{0}^{\infty} H u^{2}<\infty \text { and } \int_{0}^{\infty} H u^{2^{*}}<\infty
$$

imply that

$$
\lim _{r \rightarrow \infty} \mathcal{H}(r)=0 .
$$

But this contradicts $\mathrm{d} \mathcal{H} / \mathrm{d} r<0$ for all $r>0$. When $\lambda=\frac{1}{4} \alpha^{2},(6.9)$ can be integrated explicitly.

\section{Non-radial solutions}

In this section we show that there are cases when the least energy solutions are non-radial, proving theorem 1.4. For this, we look at the second derivative of $Q_{\lambda}$ calculated at a radial solution $u$, and we argue that there are situations when this cannot be positive semi-definite. This implies that $u$ is not a ground state. This reasoning appeared in $[13,18]$.

Proof of theorem 1.4. Let $u=u(r)$ be a radial solution of (1.2), $s \in \mathbb{R}$ and $h \in$ $H(\alpha)$. We write

$$
Q_{\lambda}(u)=\frac{\mathcal{N}(u)}{\mathcal{D}(u)},
$$

where

$$
\mathcal{N}(u)=\int K|\nabla u|^{2}-\lambda \int K|x|^{\alpha-2} u^{2}
$$


and

$$
\mathcal{D}(u)=\left(\int K u^{2^{*}}\right)^{2 / 2^{*}}
$$

(see (1.4)). Therefore,

$$
\begin{aligned}
\left.\frac{\mathrm{d}^{2}}{\mathrm{~d} s^{2}} Q_{\lambda}(u+s h)\right|_{s=0}=\frac{\left\langle\mathcal{N}^{\prime \prime}(u) h, h\right\rangle}{\mathcal{D}(u)} & -2 \frac{\mathcal{N}^{\prime}(u) h}{\mathcal{D}^{2}(u)} \mathcal{D}^{\prime}(u) h \\
& +2 \frac{\mathcal{N}(u)}{\mathcal{D}^{3}(u)}\left(\mathcal{D}^{\prime}(u) h\right)^{2}-\frac{\mathcal{N}(u)}{\mathcal{D}^{2}(u)}\left\langle\mathcal{D}^{\prime \prime}(u) h, h\right\rangle .
\end{aligned}
$$

Since by assumption $u$ is a critical point of $Q_{\lambda}$, it follows that the second and third terms cancel each other out. Therefore,

$$
\left\langle Q_{\lambda}^{\prime \prime}(u) h, h\right\rangle=\frac{\left\langle\mathcal{N}^{\prime \prime}(u) h, h\right\rangle-Q_{\lambda}(u)\left\langle\mathcal{D}^{\prime \prime}(u) h, h\right\rangle}{\mathcal{D}(u)} .
$$

Direct calculations show that

$$
\left\langle\mathcal{N}^{\prime \prime}(u) h, h\right\rangle=2 \int K|\nabla h|^{2}-2 \lambda \int K|x|^{\alpha-2} h^{2}
$$

and

$$
\begin{aligned}
\left\langle\mathcal{D}^{\prime \prime}(u) h, h\right\rangle=2\left(2-2^{*}\right)\left(\int K u^{2^{*}}\right)^{\left(2 / 2^{*}\right)-2}\left(\int K u^{2^{*}-1} h\right)^{2} & \\
+ & 2\left(2^{*}-1\right)\left(\int K u^{2^{*}}\right)^{\left(2 / 2^{*}\right)-1} \int K u^{2^{*}-2} h^{2} .
\end{aligned}
$$

Again, since $u$ is solution of (1.2), we have

$$
\mathcal{N}(u)=\int K|\nabla u|^{2}-\lambda \int K|x|^{\alpha-2} u^{2}=\int K u^{2^{*}}=\mathcal{D}(u)^{2^{*} / 2}=Q_{\lambda}(u)^{1 / 2} N .
$$

If $u$ is a local minimum of $Q_{\lambda}$, then

$$
\left\langle Q_{\lambda}^{\prime \prime}(u) h, h\right\rangle \geqslant 0
$$

for all $h \in H(\alpha)$, that is

$$
\begin{aligned}
\int K|\nabla h|^{2} & -\lambda \int K|x|^{\alpha-2} h^{2} \\
& +\frac{4}{N-2} Q_{\lambda}(u)^{-N / 2}\left(\int K u^{2^{*}-1} h\right)^{2}-\frac{N+2}{N-2} \int K u^{2^{*}-2} h^{2} \geqslant 0 .
\end{aligned}
$$

Of course, if we substitute $u$ for $h$ in the inequality above, we obtain an equality because $Q_{\lambda}$ is constant in the direction of $u$. We are therefore led to test an $h$ orthogonal to $u$. We will choose $h(x)=u(r) Y(\omega)$, where $\omega \in \mathbb{S}^{N-1}, x=r \omega$ and $Y$ is a first spherical harmonic function. That is, $Y$ is not identically zero: it satisfies

$$
-\Delta_{\omega} Y=(N-1) Y, \quad \int_{\mathbb{S}^{N-1}} Y \mathrm{~d} \omega=0
$$


we will also assume that $Y$ is normalized so that

$$
\|Y\|_{L^{2}\left(\mathbb{S}^{N-1}\right)}^{2}=1 \quad \text { and } \quad\left\|\nabla_{\omega} Y\right\|_{L^{2}\left(\mathbb{S}^{N-1}\right)}^{2}=N-1 .
$$

With this $h$ we obtain

$$
\begin{aligned}
\int_{\mathbb{R}}^{N} K|\nabla h|^{2} & =\int_{\mathbb{R}}^{N} K u_{r}^{2} Y^{2}+\int_{\mathbb{R}}^{N} K u^{2}\left|\nabla_{\omega} Y\right|^{2} \\
& =\int_{0}^{\infty} K(r) r^{N-1} u_{r}^{2} \mathrm{~d} r+(N-1) \int_{0}^{\infty} K(r) r^{N-1} u^{2} \mathrm{~d} r .
\end{aligned}
$$

Substituting this in (7.1), and since $h$ is orthogonal to all radial functions, we get

$$
\begin{aligned}
\int_{0}^{\infty} K(r) r^{N-1} u_{r}^{2} \mathrm{~d} r+ & (N-1) \int_{0}^{\infty} K(r) r^{N-1} u^{2} \mathrm{~d} r \\
& -\lambda \int_{0}^{\infty} K(r) r^{N+\alpha-3} u^{2} \mathrm{~d} r-\frac{N+2}{N-2} \int_{0}^{\infty} K(r) r^{N-1} u^{2^{*}} \geqslant 0 .
\end{aligned}
$$

Since $u$ is a radial solution of (1.2) we have

$$
\int_{0}^{\infty} K(r) r^{N-1} u_{r}^{2} \mathrm{~d} r=\lambda \int_{0}^{\infty} K(r) r^{N+\alpha-3} u^{2}+\int_{0}^{\infty} K(r) r^{N-1} u^{2^{*}} .
$$

Therefore, (7.1) implies that

$$
(N-1) \int_{0}^{\infty} K(r) r^{N-1} u^{2} \mathrm{~d} r \geqslant \frac{4}{N-2} \int_{0}^{\infty} K(r) r^{N-1} u^{2^{*}}
$$

or, equivalently,

$$
\begin{aligned}
\frac{1}{4}(N-1)(N-2) \int_{0}^{\infty} K(r) r^{N-1} u^{2} \mathrm{~d} r+\lambda \int_{0}^{\infty} K(r) r^{N+\alpha-3} u^{2} \mathrm{~d} r & \\
& \geqslant \int_{0}^{\infty} K(r) r^{N-1} u_{r}^{2} \mathrm{~d} r
\end{aligned}
$$

We will show that there exist values of the three parameters $N, \alpha$ and $\lambda$ such that the ground state is achieved and the opposite inequality to (7.2) holds. This implies that the ground state is non-radial. We already have

$$
\int_{0}^{\infty} K(r) r^{N-1} u_{r}^{2} \mathrm{~d} r \geqslant \lambda_{1} \int_{0}^{\infty} K(r) r^{N+\alpha-3} u^{2} \mathrm{~d} r .
$$

Since $u$ is assumed to be a radial solution of (1.2), it satisfies

$$
-\left(K r^{N-1} u_{r}\right)_{r}=\lambda K r^{N+\alpha-3} u+K r^{N-1} u^{2^{*}-1}, \quad u_{r}(0)=0 ;
$$

hence, $u_{r}(r)<0$ for all $r>0$. We can apply the Hardy-type inequality of lemma 7.1, below, to obtain

$$
\int_{0}^{\infty} K(r) r^{N-1} u_{r}^{2} \mathrm{~d} r \geqslant C^{-2} \int_{0}^{\infty} K(r) r^{N-1} u^{2} \mathrm{~d} r
$$


where $B \leqslant C \leqslant 2 B$ and $B$ is defined by

$$
B^{2}=\sup _{0<x<\infty} \int_{0}^{x} K(r) r^{N-1} \mathrm{~d} r \int_{x}^{\infty} \frac{1}{K(r) r^{N-1}} \mathrm{~d} r .
$$

Making the change of variables $s=\frac{1}{4} r^{\alpha}$, we can write

$$
B^{2}=\frac{4^{2 / \alpha}}{\alpha^{2}} \sup _{0<y<\infty} \int_{0}^{y} \mathrm{e}^{s} s^{\mu-1} \mathrm{~d} s \int_{y}^{\infty} \mathrm{e}^{-s} s^{-\mu+(2 / \alpha)-1} \mathrm{~d} s,
$$

where $\mu=N / \alpha$. We see that, by keeping $\mu$ relatively constant, we get $B \approx 1 / \alpha$, which is small for $\alpha$ large.

From

$$
\int_{0}^{\infty} K r^{N-1} u_{r}^{2}=\left(1-\frac{\lambda}{\lambda_{1}}\right) \int_{0}^{\infty} K r^{N-1} u_{r}^{2}+\frac{\lambda}{\lambda_{1}} \int_{0}^{\infty} K r^{N-1} u_{r}^{2}
$$

and (7.3) and (7.4) we get

$$
\int_{0}^{\infty} K r^{N-1} u_{r}^{2} \geqslant \frac{1}{4 B^{2}}\left(1-\frac{\lambda}{\lambda_{1}}\right) \int_{0}^{\infty} K r^{N-1} u^{2}+\lambda \int_{0}^{\infty} K r^{N+\alpha-3} u^{2} .
$$

Therefore, whenever

$$
\frac{1}{4 B^{2}}\left(1-\frac{\lambda}{\lambda_{1}}\right)>\frac{(N-1)(N-2)}{4},
$$

the inequality (7.2) cannot hold and so the ground state cannot be radial. Note that, in order for the ground state to exist, we have the sufficient conditions

$$
1>\frac{\lambda}{\lambda_{1}}> \begin{cases}\frac{1}{2} & \text { if } 2<\alpha \leqslant N-2, \\ \frac{\alpha^{2}}{4 \lambda_{1}} & \text { if } \alpha>N-2 .\end{cases}
$$

The lemma below is adapted from $[14,16]$.

Lemma 7.1. For every decreasing function $u=u(r)$ in $H_{\mathrm{rad}}(\alpha)$, (7.4) holds where $B \leqslant C \leqslant 2 B$, and $B$ is defined by (7.5).

Proof. Let

$$
w(r)=K(r) r^{N-1} \quad \text { and } \quad h(t)=\left(\int_{t}^{\infty} \frac{1}{w(y)} \mathrm{d} y\right)^{1 / 4} .
$$

Since $u$ is assumed decreasing, positive function, we have

$$
u(x)=\int_{x}^{\infty}\left|u^{\prime}(t)\right| \mathrm{d} t=\int_{x}^{\infty}\left|u^{\prime}(t)\right| w^{1 / 2}(t) h(t) \frac{1}{w^{1 / 2}(t) h(t)} \mathrm{d} t .
$$

The Cauchy-Schwarz inequality implies that

$$
u(x) \leqslant\left(\int_{x}^{\infty}\left|u^{\prime}(t)\right|^{2} w(t) h^{2}(t) \mathrm{d} t\right)^{1 / 2}\left(\int_{x}^{\infty} \frac{1}{w(t) h^{2}(t)} \mathrm{d} t\right)^{1 / 2} .
$$


But

$$
\begin{aligned}
\int_{x}^{\infty} \frac{1}{w(t) h^{2}(t)} \mathrm{d} t & =-2 \int_{x}^{\infty} \frac{\mathrm{d}}{\mathrm{d} t}\left(\int_{t}^{\infty} \frac{1}{w(y)} \mathrm{d} y\right)^{1 / 2} \mathrm{~d} t \\
& =2\left(\int_{x}^{\infty} \frac{1}{w(y)} \mathrm{d} y\right)^{1 / 2} \\
& =2 h^{2}(x) .
\end{aligned}
$$

Therefore,

$$
u(x) \leqslant\left(\int_{x}^{\infty} w(t)\left|u^{\prime}(t)\right|^{2} h^{2}(t) \mathrm{d} t\right)^{1 / 2} 2^{1 / 2} h(x)
$$

and

$$
\int_{0}^{\infty} w(x) u^{2}(x) \leqslant 2 \int_{0}^{\infty} w(x) h^{2}(x)\left(\int_{x}^{\infty} w(t)\left|u^{\prime}(t)\right|^{2} h^{2}(t) \mathrm{d} t\right)
$$

Changing the order of integration yields

$$
\int_{0}^{\infty} w(x) u^{2}(x) \leqslant 2 \int_{0}^{\infty}\left(\int_{0}^{t} w(x) h^{2}(x)\right) w(t)\left|u^{\prime}(t)\right|^{2} h^{2}(t) \mathrm{d} t .
$$

From the definition of $B$, we estimate that

$$
\begin{aligned}
\int_{0}^{t} w(x) h^{2}(x) & \leqslant B \int_{0}^{t} w(x)\left(\int_{0}^{x} w(y) \mathrm{d} y\right)^{-1 / 2} \\
& =2 B \int_{0}^{t} \frac{\mathrm{d}}{\mathrm{d} x}\left(\int_{0}^{x} w(y) \mathrm{d} y\right)^{1 / 2} \\
& =2 B\left(\int_{0}^{t} w(y) \mathrm{d} y\right)^{1 / 2} \\
& \leqslant 2 B^{2}\left(\int_{t}^{\infty} \frac{1}{w(y)} \mathrm{d} y\right)^{-1 / 2} \\
& =2 B^{2} h^{-2}(t)
\end{aligned}
$$

Substituting in the inequality above, we get

$$
\int_{0}^{\infty} w(x) u^{2}(x) \leqslant 4 B^{2} \int_{0}^{\infty} w(t)\left|u^{\prime}(t)\right|^{2} \mathrm{~d} t
$$

which is the inequality (7.4).

\section{Acknowledgments}

M.F. has been partly supported by FEMAT-DF and M.M. has been partly supported by CNPq. 


\section{References}

1 F. V. Atkinson and L. A. Peletier. Sur les solutions radiales de l'équation $\Delta u+\frac{1}{2} x \cdot \nabla u+$ $\frac{1}{2} \lambda u+|u|^{p-1} u=0$. C. R. Acad. Sci. Paris Sér. I 302 (1986), 99-101.

2 C. Bandle and R. Benguria. The Brezis-Nirenberg problem on $\mathbb{S}^{3}$. J. Diff. Eqns 178 (2002), 264-279.

3 C. Bandle and L. A. Peletier. Best Sobolev constants and Emden equations for the critical exponent in $\mathbb{S}^{3}$. Math. Annln 313 (1999), 83-93.

4 C. Bandle and S. Stingelin. New numerical solutions for the Brezis-Nirenberg problem on $\mathbb{S}^{n}$. In Elliptic and parabolic problems, Progress in Nonlinear Differential Equations and Their Applications, vol. 63, pp. 13-21 (Birkhäuser, 2005).

5 C. Bandle, L. A. Peletier and S. Stingelin. Best Sobolev constants and quasi-linear elliptic equations with critical growth on spheres. Math. Nachr. 278 (2005), 1388-1407.

6 H. Brezis and L. Nirenberg. Positive solutions of nonlinear elliptic equations involving critical Sobolev exponents. Commun. Pure Appl. Math. 36 (1983), 437-477.

$7 \quad$ H. Brezis and L. A. Peletier. Elliptic equations with critical exponent on $\mathbb{S}^{3}$ : new nonminimising solutions. C. R. Acad. Sci. Paris Sér. I 339 (2004), 391-394.

8 F. Catrina and R. Lavine. Radial solutions for weighted semilinear equations. Commun. Contemp. Math. 4 (2002), 529-545.

9 H. Egnell. Semilinear elliptic equations involving critical Sobolev exponents. Arch. Ration. Mech. Analysis 104 (1988), 27-56.

10 H. Egnell. Existence and non-existence results for $m$-Laplace equations involving critical Sobolev exponents. Arch. Ration. Mech. Analysis 104 (1988), 57-77.

11 M. Escobedo and O. Kavian. Variational problems related to self-similar solutions of the heat equation. Nonlin. Analysis 11 (1987), 1103-1133.

12 A. Haraux and F. Weissler. Nonuniqueness for a semilinear initial value problem. Indiana Univ. Math. J. 31 (1982), 167-189.

13 B. Kawohl. Symmetry results for functions yielding best constants in Sobolev-type inequalities. Discrete Contin. Dynam. Syst. 6 (2000), 683-690.

14 A. Kufner and L. E. Persson. Weighted inequalities of Hardy type (World Scientific, 2003).

15 N. N. Lebedev. Special functions and their applications (transl. ed. R. A. Silverman) (New York: Dover, 1972).

16 B. Opic and A. Kufner. Hardy-type inequalities. Pitman Research Notes in Mathematics Series, vol. 219 (Harlow: Longman Scientific, 1990).

17 P. Pucci and J. Serrin. Critical exponents and critical dimensions for polyharmonic operators. J. Math. Pures Appl. 69 (1990), 55-83.

18 D. Smets, M. Willem and J. Su. Non-radial ground states for the Hénon equation. Commun. Contemp. Math. 4 (2002), 467-480.

(Issued 14 December 2007) 